\title{
Interfacial correlation function for adsorption on a disc
}

\author{
P. Jakubczyk and M. Napiórkowski \\ Instytut Fizyki Teoretycznej, Uniwersytet Warszawski \\ 00-681 Warszawa, Hoża 69, Poland
}

\begin{abstract}
Within the Landau-Ginzburg-Wilson approach we derive the effective Hamiltonian governing fluctuations of an interface between two phases, one of them adsorbed on a disclike substrate. For large disc radii and for temperatures close to the wetting temperature of the corresponding planar substrate the expressions for the height-height correlation function and the accompanying correlation angle are derived. Their dependence on the disc radius and the reduced temperature is discussed and the relevant scaling regimes are identified.
\end{abstract}

PACS numbers: 68.15.+e; 68.08.Bc

Keywords: Curved interfaces, Wetting, Surface tension

Corresponding author: Paweł Jakubczyk Instytut Fizyki Teoretycznej, Uniwersytet Warszawski 0-681 Warszawa, Hoża 69, Poland; tel. 0048225532317, e-mail: Pawel.Jakubczyk@fuw.edu.pl 


\section{Introduction}

The presence of a substrate favouring one of two coexisting bulk phases may cause this phase to form a film adsorbed at its surface. At a certain temperature $T_{W}$ below the bulk critical temperature $T_{C}$ the system may undergo a wetting transition at which the thickness of the film becomes macroscopic. Such wetting transitions have been extensively studied during the last years, especially for the planar substrates; for reviews see [1-3]. In addition, a lot of effort has been made towards understanding the modifications of system's wetting behaviour introduced by structured substrates, including chemically homogeneous nonplanar substrates [4-14]. In particular, for spherical and cylindrical geometries [15-25] the substrate's finite and positive radius $R$ prevents the divergence of the adsorbed film's thickness so that wetting transition point may be reached only in the limiting case $R=\infty$. This is due to the fact that - unlike in the planar case - the area of the interface is not invariant under the change of its position - and increases with the increasing layer's thickness. The accompanying growth of the interfacial free energy prevents the wetting transition.

To our knowledge not much attention has been paid to studying interfacial correlation functions in such systems, especially from the point of view of their temperature and substrate's radius depencence [16]. Our first aim is to systematically derive the effective interfacial Hamiltonian for adsorption on a disc including its radius and temperature dependence. This Hamiltonian is then used to evaluate the interfacial correlation function and the corresponding correlation angle parametrized by $R$ and $T$. In particular, one is interested in seeing how the divergence of the correlation angle close to $T_{W}$ is prevented by finite values of $R$.

In Section 2 we introduce the Landau-Ginzburg-Wilson theory for a 2dimensional system consisting of two phases coexisting in presence of a disclike substrate. This theory serves to derive within the mean field approach the effective interface Hamiltonian. We discuss the curvature-induced modifications of its form as compared to the planar case and propose a perturbative procedure yielding approximate expression for the equilibrium interface position at a given thermodynamic state.

Section 3 contains the discussion of the correlation function for an interface fluctuating around its mean circular shape. Within the Gaussian approximation the expressions for the correlation angle and the amplitude of the correlation function are derived.

In Section 4 we apply the specific results obtained in Section 2 to evalu- 
ate the correlation function. After introducing natural scaling variables we discuss the asymptotic behaviour of the correlation length and identify the different scaling regimes. In Section 5 we summarize our results.

\section{The Hamiltonian}

The standard method of deriving the effective Hamiltonians amounts to defining a new set of variables describing the system's state on the coarse-grained scale and introducing constraints which specify a restiction on the allowed set of microstates compatible with fixed values of new variables. The effective Hamiltonian is then extracted from the logarithm of statistical sum performed under this constraint. The mean field version of this procedure amounts to minimization of the microscopic Hamiltonian under the appropriate constraint.

Our derivation of the effective Hamiltonian for a 1-dimensional interface fluctuating in presence of a disclike substrate follows the lines first outlined in Ref. 27] for the planar case. It starts from the description of a two-dimensional uniaxial ferromagnet based on the Landau-Ginzburg-Wilson Hamiltonian

$$
\mathcal{H}[m]=\int_{0}^{2 \pi} d \phi\left[\int_{R}^{\infty} r d r\left[\frac{K}{2}(\nabla m)^{2}+\Phi(m)\right]+R \Phi_{1}\left(\left.m\right|_{r=R}\right)\right]
$$

where $m(\vec{r})=m(r, \phi)$ is a scalar order parameter (magnetization), $R$ - the substrate's radius, $\Phi(m)$ and $\Phi_{1}\left(\left.m\right|_{r=R}\right)$ - the bulk and surface free energy densities. The bulk free energy density at coexistence $\Phi_{0}(m)$ exhibits two equally deep minima located at $m_{\alpha 0}<0$ and $m_{\beta 0}>0$ corresponding to two coexisting phases with opposite magnetizations. In our approach $\Phi_{0}(m)$ has the form of double parabola [26]

$$
\Phi_{0}(m)= \begin{cases}\frac{K p_{\alpha}^{2}}{2}\left(m-m_{\alpha 0}\right)^{2}, & \text { for } m \leq m^{*} \\ \frac{K p_{\beta}^{2}}{2}\left(m-m_{\beta 0}\right)^{2}, & \text { for } m \geq m^{*}\end{cases}
$$

where $K>0$, and $p_{\gamma}^{-1}=\xi_{\gamma}$ is the bulk correlation length in phase $\gamma, \gamma=$ $\alpha, \beta$. The parameter $m^{*}$ determines the value of the order parameter $m$ at which the $\beta$-phase goes over into the $\alpha$-phase, i.e., it fixes the position of the $\alpha-\beta$ interface. The choice of $m^{*}$ value is arbitrary [29], 30]; in this paper it is set equal to zero [27]. The continuity of $\Phi_{0}$ at $m=0$ implies the relation $\frac{p_{\alpha} m_{\alpha 0}}{p_{\beta} m_{\beta 0}}=-1$ between the model parameters. The double parabola 
approximation to the general $\mathrm{m}^{4}$ model makes further calculations tractable as it yields a linear equation for the constrained magnetization profile. The cost to be paid for this simplification is the necessity to limit the considered system's states to these far from bulk criticality $\left(T \ll T_{C}\right)$ (see [26], 27]). For macroscopic disc radii this corresponds to $p_{\gamma} R \gg 1$ and $\xi_{\gamma}$ not too large as compared to the surface enhancement length $\frac{K}{|g|}[19]$. The opposite case was considered in Refs[18-19].

In the presence of external magnetic field $h$ the free energy density $\Phi(m)=$ $\Phi_{0}(m)-h m$ may be rewritten as

$$
\Phi(m)= \begin{cases}\frac{K p_{\alpha}^{2}}{2}\left(m-m_{\alpha 0 h}\right)^{2}+\Phi_{\alpha} & \text { for } m \leq 0 \\ \frac{K p_{\beta}^{2}}{2}\left(m-m_{\beta 0 h}\right)^{2}+\Phi_{\beta} & \text { for } m \geq 0\end{cases}
$$

where $m_{\gamma 0 h}=m_{\gamma 0}+\frac{h}{K p_{\gamma}^{2}}$ and $\Phi_{\gamma}=-h\left(m_{\gamma 0}+\frac{h}{2 K p_{\gamma}^{2}}\right)$ are the equilibrium bulk magnetization and free energy density of phase $\gamma$, respectively. Note that for $h=0$ one has $\Phi_{\gamma}=0$. From now on we consider the thermodynamic states of the system corresponding to bulk coexistence; more precisely we set $h=0^{-}$to stabilize the $\alpha$ phase in the bulk.

The surface free energy density is reperesented by the expression

$$
\Phi_{1}\left(m_{1}\right)=-h_{1} m_{1}-\frac{1}{2} g m_{1}^{2},
$$

where $m_{1}=m(r=R, \phi), h_{1}>0$ is the surface magnetic field favouring the phase $\beta$ at the substrate, and $g$ is the surface enhancement parameter. In the present analysis we assume $g<-K p_{\beta}$ which corresponds to a second order wetting transition of a reference planar system [27].

Minimization of Eq.(2.1) is performed under the crossing constraint $m(r=$ $l(\phi), \phi)=0$, where $r=l(\phi)>R$ specifies the position of the interface. This procedure yields the Helmholtz equation for the constrained equilibrium magnetization $\widetilde{m}$

$$
K \Delta \widetilde{m}=\frac{d \Phi}{d \widetilde{m}}
$$

which has to be solved with the boundary conditions

$$
\left.K \frac{\partial \tilde{m}}{\partial r}\right|_{r=R}=\frac{d \Phi_{1}\left(\widetilde{m}_{1}\right)}{d \widetilde{m}_{1}}
$$

$\lim _{r \rightarrow \infty} \widetilde{m}\left(r, l_{0}\right)=m_{\alpha 0}$, and $\lim _{r \rightarrow l_{0}^{-}} \widetilde{m}\left(r, l_{0}\right)=\lim _{r \rightarrow l_{0}^{+}} \widetilde{m}\left(r, l_{0}\right)=0$. 


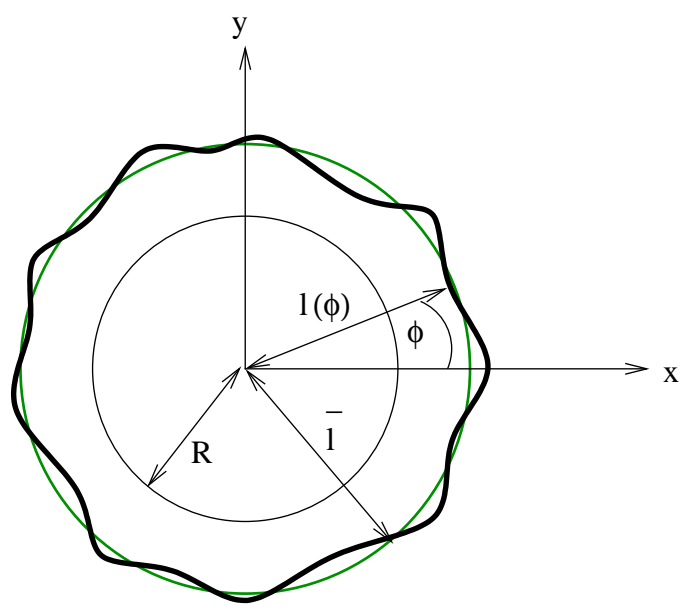

Figure 1: A schematic illustration of an interface fluctuating around its equilibrium position $\bar{l}$ in presence of a disclike substrate with radius $R$.

In the radially symmetric case, i.e., when $l=l_{0}=$ const the constrained magnetization profile $\widetilde{m}_{0}\left(r, l_{0}\right)$ fulfils the zeroth order modified Bessel equation. Its general solution has the following form

$$
\widetilde{m}_{0}\left(r, l_{0}\right)-m_{\gamma 0}=C_{\gamma} K_{0}\left(p_{\gamma} r\right)+D_{\gamma} I_{0}\left(p_{\gamma} r\right)
$$

where $K_{0}$ and $I_{0}$ are the 0 -th order modified Bessel functions, while $\gamma=\alpha$ corresponds to $r \geq l$, and $\gamma=\beta$ to $r \leq l$, respectively. The constants $C_{\gamma}$, $D_{\gamma}$ are determined from the above boundary conditions:

$$
\begin{gathered}
C_{\alpha}=\frac{-m_{\alpha 0}}{K_{0}\left(p_{\alpha} l_{0}\right)}, \quad D_{\alpha}=0 \\
C_{\beta}=\left[\frac{-m_{\beta 0}}{I_{0}\left(p_{\beta} l_{0}\right)}+F_{1}(R)\right]\left[\frac{K_{0}\left(p_{\beta} l_{0}\right)}{I_{0}\left(p_{\beta} l_{0}\right)}-F_{2}(R)\right]^{-1}, \quad D_{\beta}=-F_{1}-F_{2} C_{\beta}
\end{gathered}
$$

where

$$
\begin{gathered}
F_{1}(R)=\frac{h_{1}+g m_{\beta 0}}{K p_{\beta} I_{1}\left(p_{\beta} R\right)+g I_{0}\left(p_{\beta} R\right)}, \\
F_{2}(R)=\frac{-K p_{\beta} K_{1}\left(p_{\beta} R\right)+g K_{0}\left(p_{\beta} R\right)}{K p_{\beta} I_{1}\left(p_{\beta} R\right)+g I_{0}\left(p_{\beta} R\right)} .
\end{gathered}
$$


When the radially symmetric constrained magnetization is inserted into Eq.(2.1) one obtains - after subtracting the bulk terms - the following expression for the effective interface Hamiltonian

$$
H\left[\widetilde{m}_{0}\right]=2 \pi V\left(R, l_{0}\right)
$$

where the function $V\left(R, l_{0}\right)$ can be split into three terms

$$
V\left(R, l_{0}\right)=R \Sigma_{s \beta}(R)+l_{0} \Sigma_{\alpha \beta}\left(R, l_{0}\right)+R \omega\left(R, l_{0}\right) .
$$

The full expression for $V\left(R, l_{0}\right)$ is presented in the Appendix.

The quantity $\Sigma_{s \beta}(R)$ represents the $R$-dependent substrate-phase $\beta$ surface tension, $\Sigma_{\alpha \beta}\left(R, l_{0}\right)$ is the $R$ and $l_{0}$-dependent interfacial stiffnes coefficient, and $\omega\left(R, l_{0}\right)$ - the effective interface potential. The surface tension $\Sigma_{s \beta}(R)$ can be independently obtained by considering the disclike substrate immersed in the phase $\beta$ and the explicit expression for it is presented in the Appendix. The radially symmetric equilibrium value of $l_{0}$ will be denoted by $\bar{l}$. It minimizes the function $V\left(R, l_{0}\right)$, i.e., $\left.\frac{\partial V\left(R, l_{0}\right)}{\partial l_{0}}\right|_{l_{0}=\bar{l}}=0$.

The next step is to find the constrained magnetization profile $\widetilde{m}(r, \phi, l)$ in the case when small deviations from the radially symmetric case are present, i.e., $\widetilde{m}(r, \phi, l)=\widetilde{m}_{0}\left(r, l_{0}\right)+\delta \widetilde{m}(r, \phi, l)$ and $l(\phi)=l_{0}+\delta l(\phi)$. This can be done along the lines described in Ref. 27]. Within this approach $\delta \widetilde{m}(r, \phi, l)=\frac{\partial \widetilde{m}_{0}\left(R, l_{0}\right)}{\partial l_{0}} \delta l(\phi)\left(1+O\left(\delta l^{2}\right)\right)$. We are interested in the effective interface Hamiltonian evaluated relative to the radially symmetric equilibrium configuration in which the interface has position $r=\bar{l}$. Up to terms quadratic in $d \delta l / d \phi$ it takes the form

$$
H_{F l}[\delta l]=\int d \phi\left[\frac{1}{2} \frac{\Sigma_{\alpha \beta}(R, l)}{l}\left(\frac{d \delta l}{d \phi}\right)^{2}+V(R, l)-V(R, \bar{l})\right],
$$

while the interfacial stiffness coefficient is identified as

$$
\Sigma_{\alpha \beta}(R, l)=K l \int d r \frac{1}{r}\left(\frac{\partial \widetilde{m}_{0}(r, l)}{\partial l}\right)^{2} .
$$

To proceed analytically we limit our considerations to the case of large substrate's radius $p_{\gamma} R \gg 1$, and to such choices of the thermodynamic state that $p_{\gamma}(l-R) \gg 1$ and $(l-R) / R \ll 1$. Then the dominant contributions to the above functions take the form

$$
\begin{aligned}
\Sigma_{\alpha \beta}(R, l)=\sigma[1+ & \left.O\left(\frac{1}{p_{\alpha} l}\right)+O\left(\frac{1}{p_{\beta} l}\right)\right]+a \tau\left[1+O\left(\frac{1}{p_{\beta} R}\right)\right] \sqrt{\frac{R}{l}} e^{-p_{\beta}(l-R)}+ \\
& +\left[s_{20}\left[1+O\left(\frac{1}{p_{\beta} R}\right)\right] \frac{l^{2}}{R^{2}}-2 c\left[1+O\left(\frac{1}{p_{\beta} R}\right)\right] \frac{l}{R}(l-R)\right. \\
& \left.+2 b \tau^{2} \frac{R}{l}\left(1+O \frac{1}{p_{\beta} R}\right)\right] e^{-2 p_{\beta}(l-R)}+O\left((l-R) e^{-3 p_{\beta}(l-R)}\right)
\end{aligned}
$$




$$
\begin{gathered}
l \Sigma_{\alpha \beta}(R, l)+R \omega(R, l)=\sigma l\left[1+O\left(\frac{1}{p_{\beta} l}\right)\right]+a \tau\left[1+O\left(\frac{1}{p_{\beta} R}\right)\right] \sqrt{l R} e^{-p_{\beta}(l-R)}+ \\
\left(b \tau^{2}+\frac{c l}{R}\right)\left[1+O\left(\frac{1}{p_{\beta} R}\right)\right] e^{-2 p_{\beta}(l-R)}+O\left[\tau \sqrt{\frac{l}{R}}\left(1+O\left(\frac{1}{p_{\beta} R}\right)\right) e^{-3 p_{\beta}(l-R)}\right](2.1
\end{gathered}
$$

The coefficients $\sigma, a, b$ and $c$ are expressed in terms of the L-G-W Hamiltonian parametres:

$$
\begin{gathered}
\sigma=\frac{K}{2}\left(m_{\alpha 0}^{2} p_{\alpha}+m_{\beta 0}^{2} p_{\beta}\right), \quad a=2 K p_{\beta} m_{\beta 0}, \quad b=K p_{\beta}, \\
c=K p_{\beta} \mathcal{G} m_{\beta 0}^{2}, \quad s_{20}=\frac{1}{2} K p_{\beta}\left(\mathcal{G}^{2}+6 \mathcal{G}-1\right) m_{\beta 0}^{2}
\end{gathered}
$$

where $\mathcal{G}=-\frac{K p_{\beta}+g}{K p_{\beta}-g}$, and the parameter $\tau=\frac{h_{1}+g m_{\beta 0}}{K p_{\beta}-g}$ is interpreted as the distance from the planar substrate's wetting temperature $\left(\tau \sim \frac{T-T_{W}}{T_{W}}\right)$. The positive values $\tau>0$ correspond to the wet state of the reference planar substrate. The expressions (2.15) and (2.16) reduce to those known for the planar case (see Ref.[27]) upon neglecting terms proportional to powers of $(l-R) / R$ as compared to one.

As already remarked we are interested in system's states characterized by large values of $R$, and $T$ close $T_{W}$. In the following we shall apply mean field approximation within which the equilibrium state minimizes $V\left(R, l_{0}\right)$. Fluctuation effects missed in this kind of description are known to influence quantitatively the system's critical properties. For this reason our results will not give the correct indices in the planar limit [31]. They will also differ from those obtained in [16, where the disc wetting phenomena were discussed within transfer matrix approach but no curvature modifications of the stiffness coefficient and the potential $V$ were considered. We believe that the model discussed here correctly describes the curvature effects on the quantitative level.

Consequently, the equilibrium thickness of the adsorbed layer $\lambda=\bar{l}-R$, fulfils the following equation

$$
\begin{aligned}
\frac{\sigma}{R}+\frac{a \tau}{2 R}\left(1+\frac{\lambda}{R}\right)^{-1 / 2} e^{-p_{\beta} \lambda}-p_{\beta} a \tau\left(1+\frac{\lambda}{R}\right)^{1 / 2} e^{-p_{\beta} \lambda}+ \\
{\left[\frac{c}{R}-2 p_{\beta}\left(b \tau^{2}+c+c \frac{\lambda}{R}\right)\right] e^{-2 p_{\beta} \lambda}=0 }
\end{aligned}
$$

Its solution $\lambda$ can be determined approximately by considering the relevant case $\lambda \ll R$. The lowest order approximation $\lambda_{0}$ is obtained by neglecting in 
Eq.(2.17) all terms proportional to $\frac{\lambda}{R}$ :

$$
\frac{\sigma}{R}-p_{\beta} a \tau e^{-p_{\beta} \lambda_{0}}-2 p_{\beta}\left(b \tau^{2}+c\right) e^{-2 p_{\beta} \lambda_{0}}=0
$$

which leads to

$$
p_{\beta} \lambda_{0}=-\log \frac{-a \tau+\sqrt{a^{2} \tau^{2}+\frac{8 \sigma\left(b \tau^{2}+c\right)}{p_{\beta} R}}}{4\left(b \tau^{2}+c\right)}
$$

For $R \rightarrow \infty$ the above expression reduces to the well known solution for the planar case: $p_{\beta} \lambda_{0}(R=\infty)=-\log \frac{-a \tau}{2\left(b \tau^{2}+c\right)}$. The approximation leading to Eq.(2.19) neglects all the curvature corrections to $V(R, l)$ as compared to the planar case. To explore the curvature-induced effects we solve Eq.(2.17)

replacing the expressions $\frac{\lambda}{R}$ with $\frac{\lambda_{0}}{R}$. In this way the following equation for $\lambda_{1}$ is obtained

$$
\begin{array}{r}
\frac{\sigma}{R}+\frac{a \tau}{2 R}\left(1+\frac{\lambda_{0}}{R}\right)^{-1 / 2} e^{-p_{\beta} \lambda_{1}}-p_{\beta} a \tau\left(1+\frac{\lambda_{0}}{R}\right)^{1 / 2} e^{-p_{\beta} \lambda_{1}}+ \\
{\left[\frac{c}{R}-2 p_{\beta}\left(b \tau^{2}+c+c \frac{\lambda_{0}}{R}\right)\right] e^{-2 p_{\beta} \lambda_{1}}=0}
\end{array}
$$

with solution

$$
p_{\beta} \lambda_{1}=-\log \frac{-a \tau\left(1+\frac{\lambda_{0}}{R}\right)^{\frac{1}{2}}+\sqrt{a^{2} \tau^{2}\left(1+\frac{\lambda_{0}}{R}\right)+\frac{8 \sigma}{p_{\beta} R}\left(b \tau^{2}+c+c \frac{\lambda_{0}}{R}\right)}}{4\left(b \tau^{2}+c\left[1+\frac{\lambda_{0}}{R}\right]\right)} .
$$

By iterating this procedure one defines a sequence of approximations to $\lambda$ denoted $\lambda_{n}, n=0,1,2, \ldots$. Our analysis of the correlation function will be limited to $n=1$. Solving numerically Eq.(2.17) and comparing the numerical result with the approximate solution $\lambda_{1}$ shows that the difference $\left|\left(\lambda_{1}-\lambda\right) / \lambda\right|$ does not exceed $10^{-6}$ (see [32]).

It follows from Eq.(2.21) that the critical wetting state at which $\lambda$ diverges cannot be reached for finite $R$ values. This confirms the well known fact of non-existence of wetting transitions for surfaces with positive curvature.

\section{Correlation function}

The interfacial correlation function is defined as

$$
<\delta l\left(\phi_{1}\right) \delta l\left(\phi_{2}\right)>=\frac{\int \mathcal{D} \delta l \delta l\left(\phi_{1}\right) \delta l\left(\phi_{2}\right) e^{-\beta H_{F l}[\delta l]}}{\int \mathcal{D} \delta l e^{-\beta H_{F l}[\delta l]}} .
$$


For small gradients of the fluctuating interface and for small variations of its position relative to its equilibrium value $\delta l(\phi)=l(\phi)-\bar{l}$ one may approximate the Hamiltonian, Eq.(2.13) by the expression bilinear in $\delta l$

$$
H_{F l}[\delta l]=\frac{1}{2} \int d \phi\left[\frac{\Sigma_{\alpha \beta}(R, \bar{l})}{\bar{l}}\left(\frac{d \delta l}{d \phi}\right)^{2}+V^{\prime \prime}(R, \bar{l})(\delta l)^{2}\right],
$$

where $V^{\prime \prime}(R, \bar{l})=\left.\frac{\partial^{2} V}{\partial l^{2}}\right|_{l=\bar{l}}$.

After applying the Fourier expansion to $\delta l(\phi)$

$$
\delta l(\phi)=\sum_{n=0}^{\infty}\left[A_{n} \cos n \phi+B_{n} \sin n \phi\right]
$$

Eq.(3.2) can be rewritten as

$$
H_{F l}[\delta l]=\frac{1}{4} \sum_{n=1}^{\infty}\left(A_{n}^{2}+B_{n}^{2}\right)\left(n^{2} \frac{\Sigma_{\alpha \beta}(R, \bar{l})}{\bar{l}}+V^{\prime \prime}(R, \bar{l})\right)+\frac{1}{2} A_{0}^{2} V^{\prime \prime}(R, \bar{l})
$$

Inserting Eq.(3.4) into Eq.(3.1), and integrating over $A_{0},\left\{A_{n}, B_{n}\right\}$ leads to the following expression for correlation function

$$
<\delta l\left(\phi_{1}\right) \delta l\left(\phi_{2}\right)>=\mathcal{A}(R, \bar{l}) \frac{\cosh \left[\frac{1}{\xi_{\phi}}(1-|\Delta \phi| / \pi)\right]}{\sinh \left(\frac{1}{\xi_{\phi}}\right)}
$$

where $\Delta \phi=\phi_{1}-\phi_{2}, \quad \xi_{\phi}=\pi^{-1} \sqrt{\frac{\Sigma_{\alpha \beta}(R, \bar{l})}{V^{\prime \prime}(R, \bar{l}) \bar{l}}}$ is the correlation angle, and $\mathcal{A}=\frac{1}{\beta V^{\prime \prime}(R, \bar{l})} \frac{1}{\xi_{\phi}}$ - the correlation function's amplitude. The correlation function depends on the absolute value of the relative angle $\Delta \phi$ and is minimal at $\Delta \phi=\pi$. On the other hand, for the case $\Delta \phi=0$ one obtains $W^{2}=<\delta l\left(\phi_{1}\right) \delta l\left(\phi_{1}\right)>=\mathcal{A} \operatorname{coth}\left(1 / \xi_{\phi}\right)$. In the planar limit $R \rightarrow \infty$ the quantity $\xi_{\phi}$ corresponds to the parallel correlation length $\xi_{\|}$divided by $R$.

For further investigation of Eq.(3.5) one needs to specify $\bar{l}$ and $V^{\prime \prime}(R, \bar{l})$. In what follows we shall use the results of Section 2 by putting $\bar{l}=R+\lambda_{1}$. In this way one obtains

$$
V^{\prime \prime}(\bar{l}, R)=2 \pi\left[p_{\beta}^{2} a \tau\left(R+\frac{\lambda_{1}}{2}\right) e^{-p_{\beta} \lambda_{1}}+4 p_{\beta}^{2}\left[\left(b \tau^{2}+c\right) R+c \lambda_{1}\right] e^{-2 p_{\beta} \lambda_{1}}\right]
$$

where the higher order terms have been neglected. The quantity $\Sigma_{\alpha \beta}(R, \bar{l})$ is evaluated by substituting $\lambda_{1}$ into Eq.(2.15). 


\section{Scaling regimes}

In this section we discuss the scaling behaviour of the correlation angle $\xi_{\phi}$ and the amplitude $\mathcal{A}$ as functions of the variables $\tau$ and $R$ in the regime where $|\tau| \ll 1$ and $p_{\gamma} R \gg 1$. We introduce the dimensionless scaling variables $t$ and $r$

$$
t=\frac{a \tau}{4 c}, \quad r=\frac{a^{2} \tau^{2} p_{\beta} R}{8 \sigma c}
$$

such that Eq.(2.19) takes the following form

$$
p_{\beta} \lambda_{0}=-\log \left[-t+\sqrt{t^{2}+\frac{t^{2}}{r}}\right]
$$

Note that the variable $r$ depending both on the magnitude of $\tau$ and $1 / p_{\beta} R$ may cover arbitrary region of the positive semiaxis, whilst $p_{\gamma} R \gg 1$ implies $r / t^{2} \gg 1$.

The leading asymptotic behaviour of the correlation angle $\xi_{\phi}$ and the amplitude $\mathcal{A}$ is determined by $\lambda_{0}$. In this case one has to distinguish four regimes in the section of the $R-\tau$ plane such that $p_{\beta} R \gg 1$ and $|\tau| \ll$ 1 depending whether $T>T_{W}, T<T_{W}, r \gg 1$, and $r \ll 1$. However, introducing $\lambda_{1}$ instead of $\lambda_{0}$, as we do, modifies the correlation angle $\xi_{\phi}$ and the amplitude $\mathcal{A}$ on the level of corrections to the leading asymptotic behaviour. It turns out that from this point of view eight subsets of the asymptotic regime $p_{\beta} R \gg 1$ and $|t| \ll 1$ must be distinguished. They are listed below together with the corresponding expressions for $\lambda_{1}, \xi_{\phi}$, and $\mathcal{A}$ :

1. $T<T_{W}, r \gg 1,\left|r t^{2} \log (-2 t)\right| \ll 1$

$$
\left\{\begin{array}{l}
p_{\beta} \lambda_{1}=-\log (-2 t)\left(1+\frac{1}{4 r \log (-2 t)}+\text { H.O.T. }\right) \\
\xi_{\phi}=-\frac{1}{2 \sqrt{2}} \pi^{-3 / 2} \sqrt{\frac{2 c}{\sigma}} \frac{t}{r}\left(1-\frac{3}{8} \frac{1}{r}+\text { H.O.T. }\right) \\
\beta \sigma p_{\beta} \mathcal{A}=\frac{-\sqrt{2}}{4} \sqrt{\pi \frac{\sigma}{2 c}} \frac{1}{t}\left(1-\frac{3}{8} \frac{1}{r}+\text { H.O.T. }\right) .
\end{array}\right.
$$

2. $T<T_{W}, r \gg 1,\left|r t^{2} \log (-2 t)\right| \gg 1$

$$
\left\{\begin{array}{l}
p_{\beta} \lambda_{1}=-\log (-2 t)\left(1+\frac{1}{4 r \log (-2 t)}+\text { H.O.T. }\right) \\
\xi_{\phi}=-\frac{1}{2 \sqrt{2}} \pi^{-3 / 2} \sqrt{\frac{2 c}{\sigma}} \frac{t}{r}\left(1+k t^{2} \log (-2 t)+\text { H.O.T. }\right) \\
\beta \sigma p_{\beta} \mathcal{A}=\frac{-\sqrt{2}}{4} \sqrt{\pi \frac{\sigma}{2 c}} \frac{1}{t}\left(1+k t^{2} \log (-2 t)+\text { H.O.T. }\right),
\end{array}\right.
$$

where $k=-4 \frac{c}{\sigma}$. 
3. $T<T_{W}, r \ll 1, r^{3 / 2} \gg\left|t^{2} \log \frac{-t}{\sqrt{r}}\right|$

$$
\left\{\begin{array}{l}
p_{\beta} \lambda_{1}=-\log \frac{-t}{\sqrt{r}}\left(1+\sqrt{r} \frac{1}{\log (-t / \sqrt{r})}+\text { H.O.T. }\right) \\
\xi_{\phi}=-\frac{1}{2} \pi^{-3 / 2} \sqrt{\frac{2 c}{\sigma}} \frac{t}{\sqrt{r}}\left(1-\frac{1}{2} \sqrt{r}+\text { H.O.T. }\right) \\
\beta \sigma p_{\beta} \mathcal{A}=-\frac{1}{2} \sqrt{\pi \frac{\sigma}{2 c}} \frac{\sqrt{r}}{t}\left(1-\frac{1}{2} \sqrt{r}+\text { H.O.T. }\right) .
\end{array}\right.
$$

4. $T<T_{W}, r \ll 1, r^{3 / 2} \ll\left|t^{2} \log \frac{-t}{\sqrt{r}}\right|$

$$
\left\{\begin{array}{l}
p_{\beta} \lambda_{1}=-\log \frac{-t}{\sqrt{r}}\left(1+\frac{c}{\sigma} \frac{t^{2}}{r}+\text { H.O.T. }\right) \\
\xi_{\phi}=-\frac{1}{2} \pi^{-3 / 2} \sqrt{\frac{2 c}{\sigma}} \frac{t}{\sqrt{r}}\left(1+k \frac{t^{2}}{r} \log \frac{-t}{\sqrt{r}}+\text { H.O.T. }\right) \\
\beta \sigma p_{\beta} \mathcal{A}=-\frac{1}{2} \sqrt{\pi \frac{\sigma}{2 c}} \frac{\sqrt{r}}{t}\left(1+k \frac{t^{2}}{r} \log \frac{-t}{\sqrt{r}}+\text { H.O.T. }\right) .
\end{array}\right.
$$

5. $T>T_{W}, r \gg 1,\left|t^{2} \log \frac{t}{2 r}\right| \ll 1$

$$
\left\{\begin{array}{l}
p_{\beta} \lambda_{1}=-\log \frac{t}{2 r}\left(1-\frac{1}{2 r \log (t / 2 r)}+\text { H.O.T. }\right) \\
\xi_{\phi}=\frac{1}{\sqrt{2}} \pi^{-3 / 2} \sqrt{\frac{2 c}{\sigma} \frac{t}{\sqrt{r}}}\left(1+\frac{3 c}{\sigma} \frac{t^{2}}{r} \log \frac{t}{2 r}+\text { H.O.T. }\right) \\
\beta \sigma p_{\beta} \mathcal{A}=\frac{\sqrt{2}}{2} \sqrt{\pi \frac{\sigma}{2 c}} \frac{\sqrt{r}}{t}\left(1-\frac{2 c}{\sigma} \frac{t^{2}}{r} \log \frac{t}{2 r}+\text { H.O.T. }\right) .
\end{array}\right.
$$

6. $T>T_{W}, r \gg 1,\left|t^{2} \log \frac{t}{2 r}\right| \gg 1$

$$
\left\{\begin{array}{l}
p_{\beta} \lambda_{1}=-\log \frac{t}{2 r}\left(1-2 \frac{c}{\sigma} \frac{t^{2}}{r}+\text { H.O.T. }\right) \\
\xi_{\phi}=\frac{1}{\sqrt{2}} \pi^{-3 / 2} \sqrt{\frac{2 c}{\sigma}} \frac{t}{\sqrt{r}}\left(1+\frac{5}{2} \frac{c}{\sigma} \frac{t^{2}}{r} \log \frac{t}{2 r}+\text { H.O.T. }\right) \\
\beta \sigma p_{\beta} \mathcal{A}=\frac{\sqrt{2}}{2} \sqrt{\pi \frac{\sigma}{2 c}} \frac{\sqrt{r}}{t}\left(1+\frac{c}{2 \sigma} \frac{t^{2}}{r} \log \frac{t}{2 r}+\text { H.O.T. }\right) .
\end{array}\right.
$$

7. $T>T_{W}, r \ll 1, r^{3 / 2} \gg\left|t^{2} \log \frac{t}{\sqrt{r}}\right|$

$$
\left\{\begin{array}{l}
p_{\beta} \lambda_{1}=-\log \frac{t}{\sqrt{r}}\left(1-\frac{\sqrt{r}}{\log (t / \sqrt{r})}+\text { H.O.T. }\right) \\
\xi_{\phi}=\frac{1}{2} \pi^{-3 / 2} \sqrt{\frac{2 c}{\sigma} \frac{t}{\sqrt{r}}}\left(1+\frac{1}{2} \sqrt{r}+\text { H.O.T. }\right) \\
\beta \sigma p_{\beta} \mathcal{A}=\frac{1}{2} \sqrt{\pi \frac{\sigma}{2 c} \frac{\sqrt{r}}{t}}\left(1+\frac{1}{2} \sqrt{r}+\text { H.O.T. }\right) .
\end{array}\right.
$$

8. $T>T_{W}, r \ll 1, r^{3 / 2} \ll\left|t^{2} \log \frac{t}{\sqrt{r}}\right|$

$$
\left\{\begin{array}{l}
p_{\beta} \lambda_{1}=-\log \frac{t}{\sqrt{r}}\left(1+\frac{c}{\sigma} \frac{t^{2}}{r}+\text { H.O.T. }\right) \\
\xi_{\phi}=\frac{1}{2} \pi^{-3 / 2} \sqrt{\frac{2 c}{\sigma}} \frac{t}{\sqrt{r}}\left(1+k \frac{t^{2}}{r} \log \frac{t}{\sqrt{r}}+\text { H.O.T. }\right) \\
\beta \sigma p_{\beta} \mathcal{A}=\frac{1}{2} \sqrt{\pi \frac{\sigma}{2 c}} \frac{\sqrt{r}}{t}\left(1+k \frac{t^{2}}{r} \log \frac{t}{\sqrt{r}}+\text { H.O.T. }\right) .
\end{array}\right.
$$


In all the specified regimes of parameters $R$ and $\tau$, the quantity $\lambda_{1}$ diverges logarithmically either as function of $\tau$ or as function of $R$. In the above regimes 1 and 2 the leading term is only temperature dependent, i.e., $p_{\beta} \lambda_{1} \sim-\ln |\tau|$ whilst for the cases $3,4,7$ and 8 it depends exclusively on the radius, i.e., $p_{\beta} \lambda_{1} \sim \ln \left(p_{\beta} R\right)$. The leading contribution to the correlation angle is proportional to $\frac{1}{R \tau}$ for regimes 1 and 2 , which for the corresponding correlation length $\xi_{\|}=R \xi_{\phi}$ gives $\xi_{\|} \sim \frac{1}{|\tau|}$. On the other hand, for cases 3-8 we obtain $\xi_{\phi} \sim R^{-1 / 2}$, which means that the dominant term is temperatureindependent and converges to 0 in the limit $R \rightarrow \infty$. In these cases one has $\xi_{\|} \sim R^{1 / 2}$. For the amplitude $\mathcal{A}$ we obtain $\mathcal{A} \sim \tau^{-1}$ in cases 1,2 , and $\mathcal{A} \sim R^{1 / 2}$ in regimes $3-8$. Note that in regimes $3-8$ the interfacial width $W$ becomes proportional to $R^{1 / 4}$. We also observe that the dependence of the leading term to the derived expressions for $\xi_{\phi}$ and $\mathcal{A}$ on the free $\alpha-\beta$ interfacial stiffness coefficient $\sigma$ is not the same for all the above specified cases. In particular, at fixed $R$ and $\tau$ in regions 1 and 2 the quantity $\xi_{\phi}$ is proportional to $\sqrt{\sigma}$ while in regimes $3-8$ the leading contribution to $\xi_{\phi}$ does not depend on $\sigma$. On the other hand, as far as the dominant contribution is concerned, in regions 1 and 2 the amplitude $\mathcal{A} \sim 1 / \sqrt{\sigma}$ while in all the other cases - $\mathcal{A} \sim 1 / \sigma$.

Among the derived corrections one encounters terms which are nonanalytic in the zero curvature limit when the temperature approaches the wetting temperature of the reference planar system. (For discussion of non-analytic contributions to other thermodynamic quantities in wetting systems, see eg. Refs. 20], 21]). These terms contain a logarithm of $R$ or $\tau$ multiplied by a power of $1 / R$ or $\tau$, which is characteristic for the considered potential $V(R, l)$, appropriate for short-range interactions. In our treatment of the correlation function the presence of the non-analytic terms is a consequence of the structure of the potential $V(R, l)$ and the stiffness coefficient $\Sigma_{\alpha \beta}(R, l)$ derived in Section 2. The form of corrections to the leading asymptotic behavior of $\xi_{\phi}$ and $\mathcal{A}$ follows from the curvature-dependent potential $V(R, l)$ in regimes 1 , $3,5,6$, and 7 . In regimes 2,4 , and 8 their form follows exclusively from the necessity of incorporating the position dependence of the stiffness coefficient $\Sigma_{\alpha \beta}(R, l)$ into the analysis. To consider still the next to leading order corrections $\xi_{\phi}$ and $\mathcal{A}$ one should use - within the present approach - a better approximation to $\lambda$ (say, $\lambda_{2}$ instead of $\lambda_{1}$ ). However, additional restrictions must then be imposed on the parametres $\tau$ and $R$ to determine the character of the next correction; accounting for it causes necessity to increase the number of regimes of parametres $\tau$ and $R$ that must be distinguished. 


\section{Summary}

Starting from the Landau-Ginzburg-Wilson theory we derived within the mean field approximation the effective Hamiltonian for the fluctuations of an interface separating two coexisting phases, one of them forming a layer adsorbed on a disclike substrate. The Hamiltonian is parametrized by the temperature $T$ and the disc radius $R$, i.e., both the coefficient of the interfacial stiffness $\Sigma_{\alpha \beta}$ and the interface potential $V$ appearing in the effective Hamiltonian depend on these two parameters, and on the interface position l. We applied the Gaussian approximation and found the expression for the height-height correlation function, in particular the correlation angle $\xi_{\phi}$ and the amplitude $\mathcal{A}$. Considering the case of large disc radii and temperatures close to the wetting temperature $T_{W}$ of a reference planar system and keeping track of the position dependence of the stiffness coefficient $\Sigma_{\alpha \beta}$ and the curvature corrections to the effective interaction potential $V$ we identified the scaling regimes in the space of parametres $R$ and $T-T_{W}$ in which the dominant contributions to $\xi_{\phi}$ and $\mathcal{A}$ were determined. In addition we have derived the expression for the $R$-dependent surface tension coefficient $\Sigma_{s \beta}$ characterizing the substrate-phase $\beta$ interface. This surface tension coefficient can be represented as the power series in $1 / R$, and the corresponding Tolman's length [35, [36] turns out to be proportional to $\left(T-T_{W}\right)^{2}$.

In the limit of large disc radii our results for the equilibrium adsorbate layer thickness $\lambda$ and the correlation length $\xi_{\|} \sim R \xi_{\phi}$ differ from those obtained by Gil and Mikheev (G-M) in Ref.[16] via the transfer matrix approach. These authors' analysis referred to a temperature independent steplike potential $V_{S}(\lambda) / R=0$ for $\lambda>0, V_{S}(\lambda) / R=\infty$ for $\lambda \leq 0$ which captures the relevant features of complete wetting phenomena in the planar limit. For that case they predict $\bar{l} \sim R^{1 / 3}$ and $\xi_{\|} \sim R^{2 / 3}$. The discrepancy between our and G-M result is due to fluctuation effects missed in our description. For the same reason we fail to predict the correct values of the critical indices in the planar 2D limit 31. Performing a transfer matrix calculation taking into account the effect of curvature on the structure of the effective Hamiltonian is a demanding task. We believe that the MFT approach applied here generally accounts for the observable phenomena, though obviously the system's behaviour is further modified when fluctuations are incorporated into the description. It is worth noting that the phenomenological treatment based on balancing the curvature-induced Laplace pressure and the disjoining pressure applied by G-M to the case of long range intractions yields in the case of short range forces the results consistent with ours.

From this perspective it would be very interesting to analyze the interfa- 
cial correlation function in system with short range forces by properly taking into account all the relevant fluctuations. It has been shown [27, 33] that such planar systems may undergo either first or second order wetting depending on the system parameters. However, this alternative applies to systems with dimension larger than $d_{c}=2.41,[34$, which is not the case considered in this paper. On the other hand, whether the interfacial correlations in the case of cylinder- or spherelike substrates contain some indications of the possible change of the order of the wetting transion in the corresponding planar case remains an open question.

\section{Acknowledgment}

This work is supported by the Committee for Scientific Research via grant 2PO3B 00823.

\section{Appendix}

In this Appendix we quote the complete expressions for the effective interface Hamiltonian $V(R, l)$ in the radially symmetric case and for the substratephase $\beta$ surface tension $\Sigma_{s \beta}(R)$ as derived from the L-G-W theory. These expressions have the following form:

$$
\begin{gathered}
V(R, l)=\frac{K}{2}\left[\frac { 1 } { 2 } C _ { \beta } ^ { 2 } p _ { \beta } ^ { 2 } \left[l^{2} K_{0}\left(p_{\beta} l\right)\left(K_{0}\left(p_{\beta} l\right)-K_{2}\left(p_{\beta} l\right)\right)-R^{2} K_{0}\left(p_{\beta} R\right) \times\right.\right. \\
\left.\times\left(K_{0}\left(p_{\beta} R\right)-K_{2}\left(p_{\beta} R\right)\right)\right]+\frac{1}{2} D_{\beta}^{2} p_{\beta}^{2}\left[l^{2} I_{0}\left(p_{\beta} l\right)\left(I_{0}\left(p_{\beta} l\right)-I_{2}\left(p_{\beta} l\right)\right)-R^{2} I_{0}\left(p_{\beta} R\right) \times\right. \\
\left.\left.\times\left(I_{0}\left(p_{\beta} R\right)-I_{2}\left(p_{\beta} R\right)\right)\right]+2 C_{\beta} D_{\beta} \int_{p_{\beta} R}^{p_{\beta} l}\left[K_{0}(u) I_{0}(u)-K_{1}(u) I_{1}(u)\right] u d u\right]+ \\
+2 \pi R\left[-h_{1} m_{\beta 0 h}-\frac{1}{2} g m_{\beta 0 h}^{2}-\left(h_{1}+g m_{\beta 0 h}\right) C_{\beta} K_{0}\left(p_{\beta} R\right)-\left(h_{1}+g m_{\beta 0 h}\right) D_{\beta} I_{0}\left(p_{\beta} R\right)+\right. \\
\left.-\frac{1}{2} g C_{\beta}^{2} K_{0}^{2}\left(p_{\beta} R\right)-\frac{1}{2} g D_{\beta}^{2} I_{0}^{2}\left(p_{\beta} R\right)-g C_{\beta} D_{\beta} K_{0}\left(p_{\beta} R\right) I_{0}\left(p_{\beta} R\right)\right]+ \\
+\frac{1}{2} K \pi m_{\alpha 0 h}^{2} p_{\alpha}^{2} l^{2}\left[\frac{K_{2}\left(p_{\alpha} l\right)}{K_{0}\left(p_{\alpha} l\right)}-1\right] \quad,
\end{gathered}
$$

and

$$
\Sigma_{s \beta}(R)=-h_{1} m_{\beta 0 h}-\frac{1}{2} g m_{\beta 0 h}^{2}+
$$




$$
\begin{array}{r}
\frac{K}{4}\left(\frac{F_{1}(R)}{F_{2}(R)}\right)^{2} p_{\beta}^{2} R K_{0}\left(p_{\beta} R\right)\left[K_{2}\left(p_{\beta} R\right)-K_{0}\left(p_{\beta} R\right)\right]+ \\
-\frac{1}{2} g\left(\frac{F_{1}(R)}{F_{2}(R)}\right)^{2} K_{0}^{2}\left(p_{\beta} R\right)+\left(h_{1}+g m_{\beta 0 h}\right) \frac{F_{1}(R)}{F_{2}(R)} K_{0}\left(p_{\beta} R\right)
\end{array}
$$

where the functions $F_{1}(R)$ and $F_{2}(R)$ are defined in the main text, see Eq.(2.10). The integral $\int_{p_{\beta} R}^{p_{\beta} l}\left[K_{0}(u) I_{0}(u)-K_{1}(u) I_{1}(u)\right] d u$ in the above expression for $V(R, l)$ can be performed analytically for the case $p_{\beta} R \gg 1, p_{\beta} l \gg 1$ by applying the asymptotic expansion of the Bessel functions. The asymptotic expansion of this formula is quoted in the main text, see Eqs(2.15), (2.16).

The expression for the surface tension $\Sigma_{s \beta}(R)$ can be derived by analyzing the the L-G-W theory for a disc immersed in phase $\beta$. One notes that the function $\Sigma_{s \beta}(R)$ can be expanded in powers of $1 / p_{\beta} R$ and the first two terms of this expansion have the following form:

$$
\begin{array}{r}
\Sigma_{s \beta}(R)=\frac{K p_{\beta}}{4} \tau^{2}-h_{1}\left(m_{\beta 0}+\tau\right)-\frac{g}{2}\left(m_{\beta 0}+\tau\right)^{2}+ \\
\frac{K p_{\beta} \tau^{2}}{4} \frac{1}{p_{\beta} R}+\text { H.O.T. }
\end{array}
$$

Thus we observe that the quantity identified as the Tolman's length depends on the temperature and vanishes upon approching the planar substrate wetting temperature $T_{W}$ proportionally to $\tau^{2}$. This behavior of the Tolman's length reflects the general property of $\Sigma_{s \beta}(R)$ which can be read off from the above formula, namely that at $T=T_{W}$ the surface tension ceases depending on $R$.

Finally, within the L-G-W theory the expression $\Sigma_{\alpha \beta}(R, l)$ for the $R$ and $l$ dependent interfacial tension is identified as $\Sigma_{\alpha \beta}(R, l)=K l \int_{R}^{\infty} d r \frac{1}{r}\left(\frac{\partial \widetilde{m}(r, l)}{\partial r}\right)^{2}$. The integration can be performed in the asymptotic regime $p_{\gamma} R \gg 1$ and the result is quotes in the main text, see Eq.(2.15). 


\section{References}

[1] S. Dietrich in Phase Transitions and Critical Phenomena edited by C. Domb and J. Lebowitz (Academic Press, New York, 1988), Vol 12.

[2] M. Schick in Liquids at Interfaces, 1988, Les Houches Lectures , edited by J. Charvolin, J. F. Joanny and J. Zinn-Justin (North Holland, Amsterdam, 1990)

[3] P. G. de Gennes, Rev. Mod. Phys. 57 (1985) 827.

[4] R. R. Netz and D. Andelman, Phys. Rev. E 55 (1997) 687.

[5] H. T. Dobbs and J. M. Yeomans, Mol. Phys. 80 (1993) 877.

[6] W. R. Osborn and J. M. Yeomans, Phys. Rev. E 51 (1995) 2053.

[7] K. Rejmer and M. Napiórkowski, Z. Phys. B 102 (1997) 101.

[8] P. S. Swain and A. O. Parry, Eur. Phys. J. 4 (1998) 459.

[9] C. Rascón, A. O. Parry and A. Sartori, Phys. Rev. E 57 (1999) 5697.

[10] K. Rejmer, S. Dietrich and M. Napiórkowski, Phys. Rev. E 60 (1999) 4027.

[11] K. Rejmer and M. Napiórkowski, Phys.Rev. E 62 (2000) 588.

[12] G. P. Kubalski and M. Napiórkowski, J. Phys.: Condens. Matter 12 (2000) 9221.

[13] A. O. Parry, M. J. Greenall and A. J. Wood, J. Phys.: Condens. Matter 14(2001) 1169.

[14] G. P. Kubalski, M. Napiórkowski and K. Rejmer, J. Phys.:Condens. Matter 13 (2001) 4727.

[15] T. Bieker and S. Dietrich, Physica A 252 (1998) 85.

[16] T. Gil and L. V. Mikheev, Phys. Rev. E 52 (1995) 772.

[17] M. P. Gelfand and R. Lipowsky, Phys. Rev. B 36 (1987) 8725.

[18] J. O. Indekeu, P. J. Upton and J. M. Yeomans, Phys. Rev. Lett. 61 (1988) 2221. 
[19] P. J. Upton, J. O. Indekeu and J. M. Yeomans, Phys. Rev. B 40 (1989) 666.

[20] R. Hołyst and A. Poniewierski, Phys. Rev. B 36 (1987) 5628.

[21] R. Evans, R. Roth and P. Bryk, to be published.

[22] H. D. Wagner, J. Appl. Phys 67 (1990) 1352.

[23] P. Levinson, J. Jouffroy and F. Brochard, J. Phys. Lett. 46 (1985) L-21.

[24] I. Hadjiagapiou, J. Chem. Phys. 105 (1996) 2927.

[25] F. Brochard, J. Chem. Phys. 84 (1986) 4664.

[26] R. Lipowsky, Z. Phys. B 55 (1984) 345.

[27] A. J. Jin and M. E. Fisher, Phys. Rev. B 47 (1993) 7365.

[28] K. Rejmer and M. Napiórkowski, Z. Phys. B 97 (1995) 293.

[29] G. Backx and J. O. Indekeu, Physica A 218 (1995) 69.

[30] A. O. Parry and C. J. Boulter, J. Phys. Condens. Matter 6 (1994) 7199.

[31] D. B. Abraham in Phase Transitions and Critical Phenomena edited by C. Domb and J. Lebowitz (Academic Press, London 1986), Vol 10.

[32] The numerical check of the proposed approximation's accuracy was carried out for $\frac{a}{b}=\frac{a}{c}=\frac{a}{\sigma}=1, p_{\beta} R \in\left[10^{2}, 10^{4}\right]$ and $\tau \in[-0.5,0.5]$.

[33] A. J. Jin and M. E. Fisher, Phys. Rev. B 48 (1993) 2642.

[34] C. J. Boulter, Phys. Rev. E 57 (1998) 2062.

[35] R.C. Tolman, J. Chem. Phys. 17 (1949) 333.

[36] J.R. Henderson, Mol. Phys. 50 (1983) 741. 